\title{
LA INTERVENCIÓN DE AUTOAYUDA POR CORREO EN EL TRATAMIENTO DE FUMADORES
}

\author{
M. CARMEN MíGUEZ y ELISARDO BECOÑA \\ Universidad de Santiago de Compostela
}

(Aceptado en abril de 2003)

\begin{abstract}
En el tratamiento de los fumadores se ha incrementado en los últimos años la utilización de procedimientos para dejar de fumar que resulten accesibles a toda la población y que, al mismo tiempo, impliquen intervenciones mínimas por parte de los profesionales. En este trabajo se hace una revisión de las intervenciones de autoayuda aplicadas al tratamiento de fumadores, que utilizan materiales escritos, fundamentalmente manuales de autoayuda, y se distribuyen a través del correo. Se analiza si variables tales como la extensión, forma de entrega y/o personalización del material, contribuyen a incrementar la eficacia de tales intervenciones. Los resultados de los estudios no resultan concluyentes respecto a los beneficios de la aplicación del programa de intervención a través de manuales o folletos de autoayuda. En cuanto a la personalización del material, se evidencia como estrategia más prometedora la utilización de cartas de feedback personalizado. Futuros estudios deberían delimitar el tipo de fumadores que, con mayor probabilidad, se beneficiarían de una determinada modalidad de intervención de autoayuda.
\end{abstract}

Palabras clave: Dejar de fumar, autoayuda, intervención por correo, manual, folletos.

The self-help mail intervention for Smoker's treatment

In the treatment of the smokers has been increased in the last years the procedures for smoking cessation that result available for all smokers and that, at the same time, imply minimal interventions for the professionals. In this work is made a review of self-help interventions applied to the smoker's treatment, which use written materials, fundamentally self-help manuals, and are distributed by mail. It is analyzed if variables such as extension, delivery way and/or tailoring of the material, contribute to increase the efficacy of such interventions. The results of the studies do not come out conclusive respect to the benefits from the application of the intervention program through selfhelp manuals or booklets. About the personalising materials, there is evidence that the most promising strategy seems to be the use of personalized feedback letters. Future studies should delimit the type of smokers that, more probably, get a benefit from a specific modality of self-help intervention.

Key words: Smoking cessation, self-help, mail intervention, manual, booklets.

\section{INTRODUCCIÓN}

La mayoría de los ex-fumadores han dejado de fumar por sí mismos (Fiore, Novotny, Pierce y Giovino, 1990; Fisher, 1993). Sin embargo, existe un porcentaje elevado de fumadores que necesitan y

Correspondencia: M. Carmen Miguez, Universidad de Santiago de Compostela, Facultad de Psicología, Departamento de Psicología Clínica y Psicobiología, Campus Universitario Sur, 15782 Santiago de Compostela. Correo-e: pcmcm@usc.es. demandan ayuda para vencer la dependencia de la nicotina. La alternativa tradicional para cubrir esta necesidad la constituyen los tratamientos para dejar de fumar de tipo clínico. Sin embargo, una gran parte de los fumadores no pueden o no desean asistir a tales tratamientos (Curry, 2001)..

Así, ante un problema de la magnitud del tabaquismo, se ha intentado buscar medios de actuación que representen alternativas reales a los clásicos trata- 
mientos clínicos. A principios de los años 70, surgieron las intervenciones de autoayuda, dentro de un enfoque comunitario de intervención (Becoña y Vázquez, 1998). Las intervenciones de autoayuda, para el abandono del tabaquismo, se han considerado como un puente entre las aproximaciones clínicas y de salud pública para dejar de fumar (Curry, 1993; 2001). Se trata de un tipo de actuación en contextos naturales, cuyo principal objetivo es promocionar el autotratamiento del tabaquismo. Se denominan de autoayuda o autotratamiento porque pretenden que el fumador inicie el abandono del tabaco por sí mismo (Becoña, 1994; Lichtenstein, 1982; Lando, 1993; Schwartz, 1987; U.S.D.H.H.S., 1988). También se han utilizado otras acepciones para denominarlas: programas autoinstruccionales, de autocuidado, autoaplicados o autoadministrados e intervenciones mínimas.

La conducta de fumar ha sido una de las que más atención ha recibido en cuanto a su tratamiento mediante procedimientos de autoayuda. Los recursos de autoayuda más utilizados son las guías o manuales, los folletos, vídeos, emisiones a través de los medios de comunicación, fundamentalmente la televisión, intervenciones con ayuda del ordenador, por teléfono y por correo. Los materiales de autoayuda se pueden hacer llegar hasta los fumadores enviándolos por correo a su domicilio, mediante entregas semanales editadas en prensa, o a través de la emisión de programas por radio o televisión, en los que se va siguiendo su contenido.

La necesidad de este tipo de programas se basa en el hecho de que la prevalencia de la conducta de fumar es muy elevada (Fiore et. al., 1990, Curry, 2001), a pesar de su descenso en las últimas décadas, y no sería posible reducir la misma utilizando sólo programas clínicos. Por ello, resulta necesario, para la solución de un problema como el tabaquismo, que en la comunidad existan diferentes alternativas que se complementen. Además, algunos fumadores (p.ej., aquellos que fuman cinco o menos cigarrillos diarios), no requieren programas complejos e intensivos para modificar su conducta (Jeffery, Danaher, Killen, Farquhar y Kinnier, 1982) y, de igual manera, otros desean dejar de fumar sin acudir a un programa clínico (Cohen, Lichtenstein, Prochaska et al., 1989; Fiore et al., 1990; U.S.D.H.H.S., 1988), prefiriendo recibir ayuda a través de una intervención de autoayuda o con un formato que implique un contacto profesional mínimo (Curry, 2001; Fiore et al, 1990).

Son varias las razones por las que un fumador puede preferir un programa de autoayuda o de intervención mínima. Por ejemplo, cuando alguna dificultad le impide el poder utilizar los recursos existentes en su entorno, como la falta de tiempo, localización geográfica distante de donde tiene lugar la intervención, gastos que conlleva, etc. Las intervenciones de autoayuda presentan importantes ventajas, con respecto a las clínicas, para los participantes en las mismas: resultan más cómodas, permitiendo a los fumadores realizarlas en privado (desde su residencia habitual o lugar de trabajo), y son más baratas, ya que suponen significativamente menos tiempo profesional y recursos que los implicados en tratamientos convencionales cara a cara.

También resultan atrayentes para los profesionales, porque se pueden aplicar a un número elevado de fumadores a coste relativamente bajo. Además, pueden tener un gran impacto en la salud pública, debido a su posibilidad de distribución (Lancaster y Stead, 2002). Hay que tener en cuenta que los materiales no son caros y pueden distribuirse con facilidad, ahorrando, además, tiempo de dedicación a los participantes, pues el contacto terapéutico es mínimo. 


\section{LOS PROGRAMAS DE AUTOAYUDA CON MANUALES}

El material de autoayuda más usado y evaluado ha sido el manual de autoayuda. La mayoría de los manuales para dejar de fumar que se han evaluado son de base conductual y utilizan tratamientos multicomponentes. Generalmente se trata de una adaptación de programas clinicos, cuya efectividad ha sido comprobada, a programas con formato autoadministrado (p.ej., American Lung Association, 1984, 1986; Becoña, 1993; Danaher y Lichtenstein, 1978).

Existen una serie de ventajas potenciales de los tratamientos de autoayuda con manual, entre las que se incluye un mayor mantenimiento de los efectos del tratamiento (Glasgow, Schafer y O'Neill, 1981), lo cual es de vital importancia, puesto que la recaída constituye el principal problema de todas las conductas adictivas. Otras ventajas de la utilización de manuales son (Curry, 1993): 1) la posibilidad de ser aplicados por personal no especializado; 2) distribución a un bajo coste en una variedad de contextos; 3) se pueden elaborar para diferentes poblaciones diana (p.ej., basándose en el estadio de cambio o en características específicas de los fumadores); 4) los fumadores pueden adaptar el programa a sus necesidades específicas (por ejemplo, variar el orden en que se realicen los ejercicios recomendados), pueden seguirlo a su ritmo y terminarlo más tarde; 5) por la posibilidad de guardar los materiales escritos, los fumadores, que fracasan en su intento de abandono del tabaco, pueden repetir con facilidad el programa (Glynn, Boyd y Gruman, 1990a) y, en caso de haber conseguido la abstinencia y experimentar momentos de tentación o consumos puntuales, se pueden utilizar para trabajar la motivación y reafirmarse en la decisión de mantenerse abstinente.

Además, si enfocamos el cambio de la conducta de fumar tomando como referencia los estadios de cambio (Prochaska y Prochaska, 1993), las intervenciones mínimas que utilicen un manual pueden contribuir a que los fumadores avancen en el estadio de cambio en el que se encuentran. De este modo, es posible que los sujetos que no se han concienciado de su problema, lo hagan $y$, los que quieren dejar de fumar, lo logren. Ello puede desempeñar un papel importante, especialmente entre los fumadores de alto riesgo, que son los que menos acuden a tratamiento.

Sin embargo, al igual que otros procedimientos de intervención, los manuales de autoayuda también tienen sus limitaciones o desventajas. Su sencillez, la economía de aplicación y posibilidad de adecuarse a cualquier persona y contexto, no siempre conllevan que la utilización del manual suponga una ventaja. De hecho, el que sean tan fácilmente aplicables, puede resultar en algunas ocasiones un inconveniente, puesto que deja la implantación del programa al arbitrio del sujeto, el cual ha de tomar decisiones en torno a las diversas cuestiones que surgen a lo largo del proceso de dejar de fumar, implicándole una mayor responsabilidad personal. Esto conlleva el riesgo de que el sujeto no aplique el programa correctamente (Brown y Owen, 1992). A su vez, aunque todas las guías o manuales pretenden eliminar el consumo de tabaco, suelen proponer un cambio general en otros hábitos relacionados con la salud (actividad física, alimentación, consumo de alcohol, etc). Esto, que en un principio puede resultar muy positivo, también puede representar un factor desmotivante para aquellos que sólo deseen dejar de fumar, sin cambiar otros aspectos de su vida.

Otra limitación que presentan los métodos de autoayuda estándar, editados en forma de manuales, es el no tener en cuenta las peculiaridades de la conducta 
de fumar de cada persona (por ejemplo, su consumo de cigarrillos diario), ni permitir una adaptación del procedimiento al progreso que vaya obteniendo cada cual.

A pesar de las limitaciones comentadas, en los últimos años estas intervenciones han cobrado una gran relevancia, como alternativa de tratamiento para que los fumadores dejen de fumar, aunque cuando se utilizan los materiales de autoayuda por si solos, generalmente, su eficacia resulta inferior a la obtenida con programas clínicos. De igual manera, se ha encontrado que cuando se obtienen porcentajes de abstinencia bajos, éstos se correlacionan con distintas variables. Entre ellas, se encuentran la cantidad de materiales leídos y el grado de adherencia a las actividades del programa de intervención (Cummings, Emont, Jaen y Sciandra, 1988; Davis, Faust y Ordentlich, 1984; Glasgow et al., 1981; Míguez, Vázquez y Becoña, 2000). Esto es, un elevado porcentaje de los fumadores que solicitan estos materiales fracasan a la hora de completar muchas de las actividades recomendadas.

Algunos de los factores que se ha visto que dificultan la adherencia son la extensión de algunos manuales (Strecher, Kreuter, Den Boer et al., 1994) y la complejidad de sus tareas (Glasgow et al., 1981), siendo necesario un nivel de lectura elevado para poder seguirlos (O’Farrell y Keuthen, 1983). Esto ha propiciado la elaboración de materiales más sencillos, tales como folletos, que se distribuyen por correo semanalmente (p.ej., Becoña y Vázquez, 2001a).

Aunque las intervenciones de autoayuda generales o estándar no consiguen porcentajes de abstinencia demasiado elevados (Shiffman, Paty, Rohay, Di Marino y Gitchell, 2000), en diferentes estudios se ha comprobado que si se incluyen intervenciones complementarias a los materiales de autoayuda, tales como lla- madas telefónicas (p.ej., Brown, Hunt y Owen, 1992; Míguez, Vázquez y Becoña, 2002; Orleans, Schoenbach, Wagner et al., 1991) o feedback personalizado (p.ej., Becoña y Vázquez, 2001b), mejoran significativamente los resultados, sobre todo entre los fumadores que buscan tratamiento.

Otra alternativa que se ha utilizado, con la finalidad de incrementar la eficacia de los materiales escritos, es adaptar estos materiales (variando aspectos tales como su extensión y/o contenido), teniendo en cuenta distintas variables de la población a la que van dirigidos, facilitando con ello su comprensión y aceptación. Varios expertos han indicado que los manuales para dejar de fumar deberían adaptarse a las características sociodemográficas, culturales y relacionadas con fumar de los fumadores (p.ej., Strecher, Rimer y Monaco, 1989; Glynn et al., 1990a,1990b), pues ello contribuiría a lograr porcentajes de abstinencia más altos que con los manuales estándar. Esto ha derivado en la realización de estudios utilizando la adaptación de los materiales, a través de distintos procedimientos, a poblaciones específicas de fumadores. Así, se han desarrollado manuales para fumadores en los diferentes estadios de preparación para el abandono (p.ej., Prochaska, DiClemente, Velicer y Rossi, 1993), teniendo en cuenta la edad (p.ej., Rimer, Orleans, Fleisher et al., 1994), estado de gestación (p.ej., Windsor, Lowe, Perkins et al., 1993) y nivel de comprensión lectora (p.ej., Orleans et al., 1991), pero los datos obtenidos al respecto no resultan del todo concluyentes. Así, por ejemplo, Windsor et al. (1993) encontraron que una guía de autoayuda adaptada a mujeres embarazadas era más efectiva que una guía genérica, al igual que en el estudio de Rimer et al. (1994), en el que una guía adaptada a personas mayores fumadoras resultaba más efectiva, a los 12 meses, que la utilizada como 
control. Sin embargo, los resultados de otros estudios tienden a apoyar la conclusión de que la mayoría de las guías contienen básicamente la misma información y que el adaptarlas no parece condicionar porcentajes de abstinencia superiores (p.ej., Brown y Owen, 1992; Cummings, Sciandra, Davis y Rimer, 1989; Davis, Cummings, Rimer, Sciandra, Stone, 1992). Además, se ha observado que la existencia de pequeñas diferencias en el contenido del programa de intervención no afectan al resultado; algo también encontrado con programas clínicos cara a cara más intensivos (Lichtenstein y Glasgow, 1992).

En relación a los materiales adaptados a los diferentes estadios de cambio, destacar que los estudios que los utilizan no se plantean como objetivo prioritario el logro de la abstinencia, sino el avanzar en el estadio de cambio (p.ej., Dijkstra, De Vries, Roijackers y Breukelen, 1998a), pues suelen estar destinados a los fumadores menos motivados (p.ej., Dijkstra, De Vries, Roijackers y Breukelen, 1998b).

Cuando se trata de personalizar la intervención, adaptándola a cada fumador, se suelen utilizar cartas que proporcionan feedback con refuerzo y consejos, en función del progreso o dificultades que vaya teniendo cada cual a lo largo del proceso de abandono. Este tipo de recursos, adaptados y personalizados, se considera que pueden promover la adherencia al tratamiento (p.ej., Curry, Wagner y Grothaus, 1991) o mejorar la realización de la intervención (p.ej., Becoña y Vázquez, 2001b) y, por lo tanto, aumentar su eficacia.

Por su relevancia, a continuación tratamos de analizar si características de los materiales de autoayuda impresos, tales como su extensión, forma de entrega y/o personalización, contribuyen a incrementar la eficacia de las intervenciones de autoayuda por correo para dejar de fumar.

\section{INTERVENCIONES DE AUTOAYUDA CON MATERIALES RECIBIDOS POR CORREO}

Es a partir de los años 80 cuando empiezan a generalizarse las investigaciones con materiales de autoayuda. Revisando el PsycLIT y MEDLINE, de 1980 a 2002, aparecen 56 estudios de autoayuda. Sin embargo, éstos se reducen considerablemente si tenemos en cuenta sólo aquellos que cumplen los criterios de inclusión que hemos utilizado para seleccionar los estudios: investigaciones en las que se utilizaron ensayos controlados aleatorizados, con materiales de autoayuda impresos recibidos por correo, y con seguimientos de al menos seis meses. Además, no se incluyeron aquellos cuyo objetivo era distinto a dejar de fumar como, por ejemplo, disminuir el consumo o avanzar en el estadio de cambio.

Los estudios que se muestran en la Tabla 1 utilizan intervenciones de autoayuda, con manuales y/o folletos para dejar de fumar recibidos por correo, y estudian el efecto de variables como la extensión y/o forma de entrega del material escrito. En 1984, Davis, Faust y Ordentlich (1984) publicaron una investigación en la que se evaluaba un manual de abandono del tabaco y otro de mantenimiento, desarrollados ambos por la Asociación Americana del Pulmón. La muestra, formada por 1.237 fumadores de la población general, se esignó al azar a uno de los cuatro grupos experimentales siguientes: 1) ocho folletos (2 dirigidos al abandono del tabaco y 6 informativos, sobre temas relacionados con el mismo) de la Asociación Americana del Pulmón ( $\mathrm{n}=308$ ); 2) los ocho folletos anteriores y el manual de mantenimiento titulado "Una vida libre de tabaco" (A lifetime of freedom from smoking) $(\mathrm{n}=312) ; 3)$ el manual de abandono *Libre del tabaco en 20 días" (Freedom from Smoking in 20 days) ( $n=308) ; y, 4)$ el manual de 
Tabla 1. Intervenciones de autoayuda con materiales de diferente extensión y/o forma de entrega

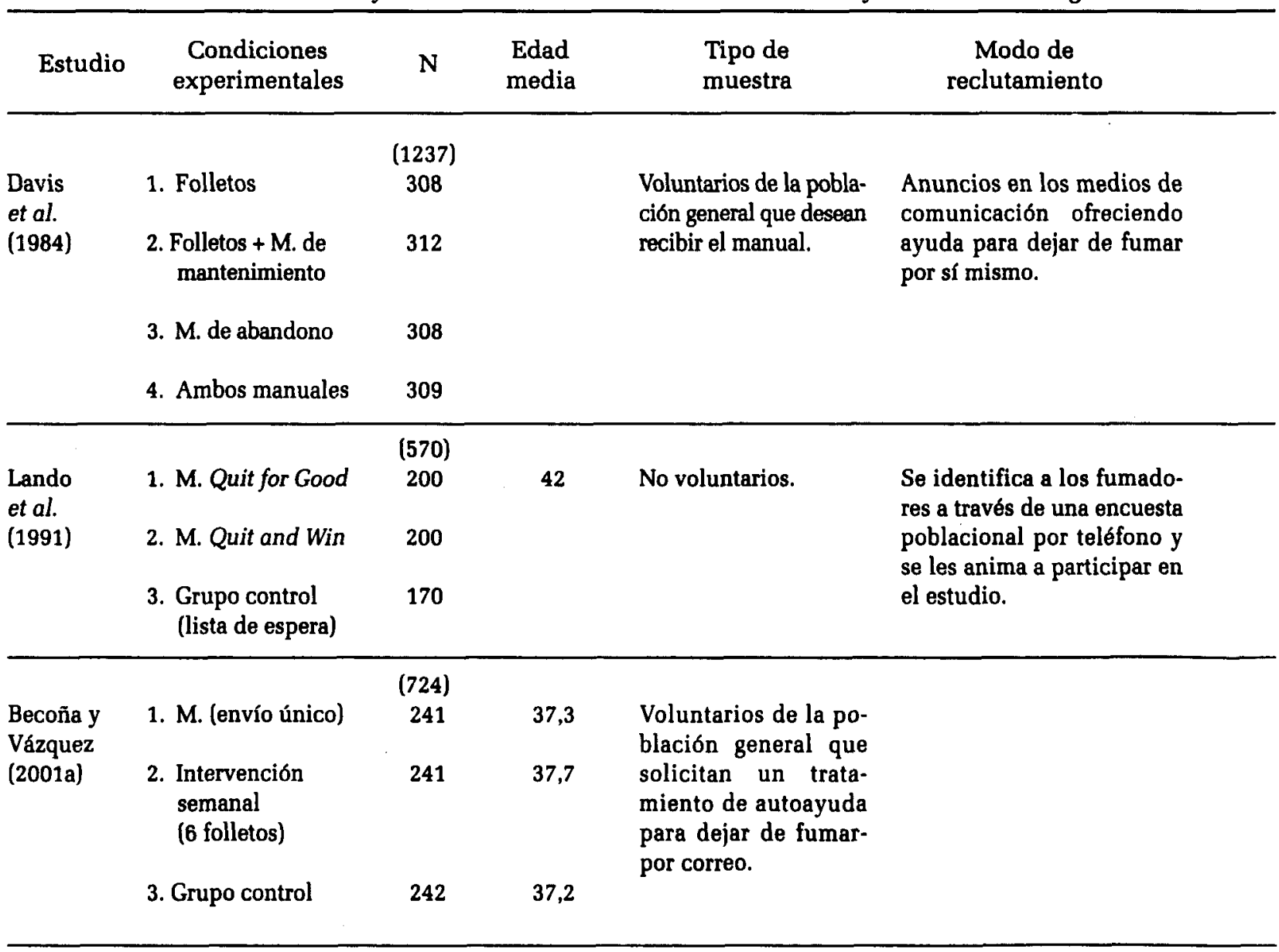

Notas: $\mathrm{M} .=$ manual de autoayuda; $\mathrm{b}$ = un mes o más de abstinencia; $\mathrm{c}$ = abstinencia continua.

abandono y el manual de mantenimiento anteriores $(\mathrm{n}=309)$.

Los seguimientos se realizaron, por teléfono y por correo, a los $1,3,6,9$ y 12 meses después de haber enviado los materiales. A los participantes no localizados se les consideró fumadores. Se utilizaron tres definiciones de no fumador: haber conseguido el abandono de los cigarrillos después del envío del material, no haber fumado durante el último mes y abstinencia continua desde el final del tratamiento.

Los grupos con mejor y peor costeefectividad fueron los que incluyeron el manual de mantenimiento y el que utilizó folletos, respectivamente. Como pue- de verse en la Tabla 1, el grupo de folletos sin manual mostró la menor eficacia en todos los seguimientos. Los autores justifican los bajos porcentajes de abstinencia del estudio por los criterios tan estrictos de abstinencia que utilizaron.

Destaca que en todos los grupos se observaron porcentajes de prevalencia de abstinencia crecientes, lo que sugiere que los intentos de abandono exitosos se incrementaron con el tiempo. Además, este estudio muestra que un componente de mantenimiento, añadido a unos sencillos folletos de autoayuda, produce porcentajes más altos de abstinencia que los folletos o el manual de abandono solo. En este caso, el manual de mantenimien- 


\begin{tabular}{|c|c|c|c|c|c|c|}
\hline \multirow{2}{*}{$\begin{array}{l}\text { Criterios de } \\
\text { admisión }\end{array}$} & \multirow{2}{*}{$\begin{array}{c}\text { Cigar./día } \\
\text { antes del } \\
\text { tratamiento }\end{array}$} & \multicolumn{4}{|c|}{$\begin{array}{l}\text { PORCENTAJE DE ABSTINENCIA } \\
\text { SEGUIMIENTOS }\end{array}$} & \multirow{2}{*}{$\begin{array}{c}\text { Validación } \\
\text { de la absti- } \\
\text { nencia }\end{array}$} \\
\hline & & 3 meses & 6 meses & 12 meses & otros & \\
\hline \multirow[t]{2}{*}{$\begin{array}{l}\text { - Firmar un consentimiento. } \\
\text { - Rellenar un cuestionario. } \\
\text { - Hacer un depósito. } \\
\text { - Participar en } 5 \text { segui- } \\
\text { mientos hasta un año }\end{array}$} & $\begin{array}{l}29,9 \\
29,0 \\
30,6 \\
29,4 \\
\\
30,6\end{array}$ & $\begin{array}{c}5 / 4 \mathrm{c} \\
9 / 5 \mathrm{c} \\
11 / 6 \mathrm{c} \\
12 / 8 \mathrm{c}\end{array}$ & $\begin{array}{c}8 b / 3 c \\
10 b / 4 c \\
10 b / 4 c \\
13 b / 6 c\end{array}$ & $\begin{array}{l}12 \mathrm{~b} / 2 \mathrm{c} \\
18 \mathrm{~b} / 4 \mathrm{c} \\
15 \mathrm{~b} / 3 \mathrm{c} \\
18 \mathrm{~b} / 5 \mathrm{c}\end{array}$ & $\begin{array}{c}\text { final } \\
16 \\
25 \\
20 \\
\\
20\end{array}$ & Autoinforme \\
\hline & 20,0 & & & & $\begin{array}{c}7 \text { meses } \\
10,2 \\
8,9 \\
10,0\end{array}$ & Autoinforme \\
\hline $\begin{array}{l}\text { - Residir en Galicia. } \\
\text { - Fumar mínimo } 10 \text { cigar./ } \\
\text { día. } \\
\text { - Cumplimentar cuestio- } \\
\text { narios. } \\
\text { - Enviar autoevaluación. } \\
\text { Participar en segui- } \\
\text { mientos. }\end{array}$ & $\begin{array}{l}26,1 \\
26,5\end{array}$ & $\begin{array}{l}22,8 \\
31,5\end{array}$ & 25,3 & $\begin{array}{l}23,2 \\
26,6\end{array}$ & $\begin{array}{l}\text { final } \\
35,3 \\
36,9\end{array}$ & $\begin{array}{l}\text { Autoinforme y } \\
\text { CO (a los } 12 \\
\text { meses) }\end{array}$ \\
\hline
\end{tabular}

to parece ser más importante para el éxito que el propio programa dirigido al abandono del tabaco.

Con frecuencia, las intervenciones para dejar de fumar por correo están dirigidas a muestras de voluntarios que solicitaron ayuda. Estos voluntarios pueden no ser representativos de la población total de fumadores. Por ello, un avance desde la perspectiva de la salud pública implica llegar a aquellos fumadores que no se presentan por sí mismos para el tratamiento. Lando, Pirie, McGovern et al. (1991) evaluaron el uso de materiales de autoayuda en población general de la ciudad de Minnesota. Utilizaron dos manuales: «Déjalo por tu bien» (Quit for good) (National Cancer Institute, 1985) y "Déjalo y gana» (Quit and Win) (Pechacek, Arkin y Hall, 1984), elaborado para este proyecto. Los sujetos de este estudio eran aquellos que fueron identificados como fumadores cuando participaron en un cribaje de factores de riesgo cardiovasculares a nivel comunitario. La muestra la formaban $\mathbf{5 7 0}$ fumadores que aceptaron participar en el programa. Los sujetos se asignaron al azar a una de las tres condiciones experimentales siguientes: 1) manual Quit for Good ( $\mathrm{n}=200)$; 2) manual Quit and Win ( $n=200)$; y 3) grupo control de lista de espera $(n=170)$.

Se planteó que el programa Quit and Win, por ser más estructurado y conside- 
rablemente más extenso, lo cual permite una discusión más detallada de estrategias de afrontamiento específicas, produciría mayor número de intentos de abstinencia que el material del National Cancer Institute y que el grupo control. Hay que tener en cuenta que aunque los materiales del Quit and Win parecen más atractivos que los del Quit for Good, su mayor longitud y detalles podrían ser un problema, especialmente en un grupo de sujetos menos motivado.

El seguimiento se hizo por teléfono siete meses después de la entrega de los manuales; los que no contestaban la entrevista eran considerados como fumadores. Los resultados se obtuvieron a través de autoinforme y no se validaron bioquímicamente. En contra de las expectativas, a los 7 meses de seguimiento no hubo diferencias significativas entre los grupos, incluso con el grupo control que no recibió materiales. Hay que tener en cuenta que, casi la mitad de los sujetos, dejaron de fumar antes de que les llegase por correo el manual y después de la entrevista por teléfono preguntándoles si querían participar en el programa. En este caso, la llamada pudo haber sido un importante estímulo para el abandono del tabaco.

En España, Becoña y Vázquez (2001a) evaluaron la eficacia de una intervención de autoayuda para dejar de fumar administrada a través del correo de dos formas: en envío único (manual de autoayuda) y en varios envíos (6 folletos enviados semanalmente). La muestra estaba formada por 724 fumadores, asignados al azar a las siguientes condiciones: 1) manual ( $\mathrm{n}=241) ; 2$ ) folletos ( $\mathrm{n}=$ 241); y 3) grupo control de lista de espera $(n=242)$. El estatus de fumador se evaluó, a través de carta o teléfono, al final del tratamiento y a los 3,6 y 12 meses de seguimiento. A los 12 meses se verificaron bioquímicamente los autoinformes de abstinencia con la medida de monóxi- do de carbono en aire espirado. Al final del tratamiento, la abstinencia fue del $35.3 \%$ para la intervención de envío único, $36.9 \%$ para la condición semanal y $\mathbf{0 . 8 \%}$ para el grupo control. A los 3 meses, los resultados fueron del $22.8 \%$, $31.5 \%$ y $2.5 \%$, y a los 6 meses, la abstinencia fue de $24.1 \%, 25.3 \%$ y $2.5 \%$, respectivamente. A los 12 meses de seguimiento, $23.2 \%$ estaban abstinentes en la intervención de envío único y $26.6 \%$ en la semanal. La intervención semanal obtuvo mejores resultados que el envío del manual en todos los momentos temporales, aunque las diferencias sólo fueron estadísticamente significativas a los 3 meses de seguimiento. Por lo tanto, en este estudio, las dos aproximaciones de correo resultaron ser igualmente beneficiosas y equivalentes.

\section{INTERVENCIONES DE AUTOAYUDA PERSONALIZADAS}

Una de las principales críticas planteadas a las intervenciones de autoayuda por correo para dejar de fumar es su impersonalización, pues, habitualmente, utilizan manuales estándar. A fin de mejorar sus resultados, se ha tratado de adaptar el tratamiento a las características individuales del fumador y a sus progresos, personalizando las instrucciones dadas a éste. Se trata de proporcionar algunas de las ventajas de las intervenciones clínicas, $\sin$ que resulte necesario asistir a sesiones de tratamiento $y$, para ello, se ha utilizado el ordenador de diferentes formas. Una modalidad de utilización del ordenador, es la empleada por Schneider, Benya y Singer (1984), a través de un programa denominado Smoke. Se trata de un programa de tipo conductual que utilizaba, en aquel momento de forma novedosa, el correo electrónico para tratar a fumadores que no deseaban asistir a un programa formal cara a cara para dejar 
de fumar. En este estudio participaron un total de $\mathbf{1 . 0 4 4}$ fumadores. Se les daba a elegir entre un tratamiento por correo o un tratamiento en grupo de cinco sesiones. Se compararon cuatro condiciones: 1) 117 prefirieron el tratamiento formal, asignándose al azar el resto de los sujetos, a cada una de las siguientes condiciones de autoayuda: 2) correo interactivo (electrónico) $(n=509), 3)$ manual enviado por correo en un único envío $(n=214)$ y, 4) folletos enviados por correo ( $n=205)$.

El grupo de correo electrónico recibió tratamiento conductual orientado a alcanzar la abstinencia en cuatro semanas. En el primer mensaje, que los sujetos recibían en una terminal de ordenador, se les instruía en el modo de registrar la conducta y se les aplicaba un cuestionario sobre el consumo de tabaco y sobre la posibilidad de que alguna persona de su entorno les ofreciese apoyo. Se les pedía que estableciesen una fecha fija para dejar de fumar, alrededor de cuatro semanas más tarde. Los participantes rellenaban los cuestionarios y los enviaban en un sobre, contestándoseles con otra carta mediante el correo electrónico, en función de las respuestas de cada fumador. Las interacciones continuaron dos semanas después de alcanzada la abstinencia. En el grupo de correo, los fumadores recibieron un manual con un procedimiento similar al del grupo anterior, pero sin el aspecto interactivo. El cuarto grupo recibió, en un único envío, distintos folletos dirigidos al abandono del tabaco.

El seguimiento se realizó por carta 1,2 y 6 meses después de comenzar el tratamiento. Los sujetos debían remitir por correo muestras de saliva para validar la abstinencia con la medida de tiocianato. Los no localizados se computaron como fumadores. En el grupo de correo electrónico y en el correspondiente al tratamiento formal, sólo se incluyeron para los análisis a aquellos fumadores que habian completado el tratamiento.

Los porcentajes de abstinencia un mes después del inicio del tratamiento fueron del $\mathbf{2 9 . 5 \%}$ en el grupo con tratamiento cara a cara, $21.3 \%$ para el de correo electrónico, del $3.2 \%$ en el de manual por correo y del $5.9 \%$ en el grupo con folletos. La condición de correo electrónico y de cara a cara obtuvo porcentajes similares, sin diferencias significativas entre ambos grupos. A los seis meses, la abstinencia fue del $18.1 \%, 12.9 \%, 9.8 \%$ y $12.3 \%$, respectivamente. Este porcentaje de abstinencia del $12.9 \%$ es interesante si tenemos en cuenta que el coste de este programa es reducido, en comparación con un programa de corte individual del mismo alcance. La abstinencia continua durante los seis meses fue de $\mathbf{1 4 . 3 \%}$, $7.4 \%, 1.4 \%$ y $1.9 \%$, respectivamente. Sin embargo, si consideramos fumadores a los sujetos que no completaron el tratamiento, tanto en el grupo formal como en el que recibe correo interactivo, la abstinencia puntual a los 6 meses pasaría a ser de $16 \%$ y $2.6 \%$, respectivamente. Destacar que la condición de correo electrónico obtuvo sus mejores resultados con fumadores ligeros.

Owen, Ewins y Lee (1989) realizaron una comparación de dos formatos de una intervención por correo para dejar de fumar, estándar y personalizada. Se evaluaron los efectos del tratamiento por correo, diseñado para proporcionar cantidades de información pequeñas y manejables, y el impacto de ajustar la intervención. La muestra que utilizaron estuvo formada por 208 fumadores que se asignaron a tres condiciones: 1 ) condición control "quit kit» (programa para dejar de fumar en cinco días) $(n=40) ; 2)$ curso por correo estándar $(n=86) ; y, 3)$ curso por correo personalizado $(n=82)$. A los sujetos de la condición control se les devolvió la cuota del curso, junto con una carta explicativa indicando que debi- 
Tabla 2. Intervenciones de autoayuda que evalúan tratamientos personalizados vs. estándar

\begin{tabular}{|c|c|c|c|c|c|}
\hline Estudio & $\begin{array}{l}\text { Condiciones } \\
\text { experimentales }\end{array}$ & $\mathrm{N}$ & $\begin{array}{l}\text { Edad } \\
\text { media }\end{array}$ & $\begin{array}{l}\text { Tipo de } \\
\text { muestra }\end{array}$ & $\begin{array}{l}\text { Modo de } \\
\text { reclutamiento }\end{array}$ \\
\hline $\begin{array}{l}\text { Schneider } \\
\text { et al. } \\
(1984)\end{array}$ & $\begin{array}{l}\text { 1. Tratamiento for- } \\
\text { mal } \\
\text { 2. Correo electrónico } \\
\text { interactivo } \\
\text { 3. M. enviado por } \\
\text { correo } \\
\text { 4. Folletos enviados } \\
\text { por correo }\end{array}$ & $\begin{array}{c}(1044) \\
117 \\
509 \\
214 \\
204\end{array}$ & & $\begin{array}{l}\text { Fumadores que acu- } \\
\text { dían al hospital de vete- } \\
\text { ranos de Nueva York. }\end{array}$ & \\
\hline $\begin{array}{l}\text { Owen } \\
\text { et al. } \\
(1989)\end{array}$ & $\begin{array}{l}\text { 1. Tratamiento están- } \\
\text { dar } \\
\text { 2. Tratamiento per- } \\
\text { sonalizado } \\
\text { 3. Condición control } \\
\text { "Quit kit» }\end{array}$ & $\begin{array}{r}(208) \\
86 \\
82 \\
40\end{array}$ & 42 & $\begin{array}{l}\text { Voluntarios población } \\
\text { general (que desean } \\
\text { dejar de fumar con un } \\
\text { programa por correo) }\end{array}$ & $\begin{array}{l}\text { Anuncios en la radio local, } \\
\text { televisión y prensa. }\end{array}$ \\
\hline $\begin{array}{l}\text { Becoña y } \\
\text { Vázquez } \\
\text { (2001b) }\end{array}$ & $\begin{array}{l}\text { 1. M. estándar } \\
\text { 2. M. estándar + } \\
\text { feedback escrito } \\
\text { personalizado }\end{array}$ & $\begin{array}{c}(300) \\
100 \\
100\end{array}$ & $\begin{array}{l}36,5 \\
36,7 \\
\\
37,9\end{array}$ & $\begin{array}{l}\text { Voluntarios que solici- } \\
\text { tan un programa de } \\
\text { autoayuda para dejar } \\
\text { de fumar por correo. }\end{array}$ & $\begin{array}{l}\text { Anuncios en prensa, radio y } \\
\text { televisión local. }\end{array}$ \\
\hline & 2. Grupo control & 100 & 35,9 & & \\
\hline
\end{tabular}

Notas: M. = manual de autoayuda.

do al elevado número de solicitudes recibidas no se les podía ofrecer el curso. Se les enviaba un "quit kit» y se les animaba a intentarlo por su cuenta. Este material incluía un plan para dejarlo en cinco días. El material del "quit kit» era similar al del curso por correo, pero más breve.

El curso estándar se componfa de cuatro lecciones enviadas semanalmente. La técnica de tratamiento recomendada era reducir a la mitad el consumo diario de cigarrillos durante los tres días anteriores al momento elegido para el abandono del tabaco. El curso personalizado utilizó el mismo contenido, secuencia de lecciones y programa de envío que el curso estándar. Las lecciones eran hechas para cada individuo usando un ordenador. Los contenidos de los párrafos insertados dentro del texto de cada lección del curso estándar estaban determinados por la información recogida en el formulario de registro. Se hipotetizó que ambas versiones del curso por correo podrían resultar en un porcentaje más alto de abstinencia que el que conseguiría el "quit kit", y que el curso personalizado tendría más impacto en la conducta de fumar que el estándar. 


\begin{tabular}{|c|c|c|c|c|c|c|}
\hline \multirow[t]{3}{*}{$\begin{array}{l}\text { Criterios de } \\
\text { admisión }\end{array}$} & \multirow[t]{2}{*}{$\begin{array}{c}\text { Cigar./día } \\
\text { antes del } \\
\text { tratamiento }\end{array}$} & \multicolumn{4}{|c|}{$\begin{array}{l}\text { PORCENTAJE DE ABSTINENCIA } \\
\text { SEGUIMIENTOS }\end{array}$} & $\begin{array}{c}\text { Validación } \\
\text { de la absti- } \\
\text { nencia }\end{array}$ \\
\hline & & & $\begin{array}{r}16,0 \\
2,6 \\
9,8 \\
12,3\end{array}$ & & $\begin{array}{c}1 \text { mes } \\
29,3 \\
21,3 \\
3,2 \\
5,9\end{array}$ & $\begin{array}{l}\text { Autoinforme y } \\
\text { tiocianato } \\
\text { (saliva) }\end{array}$ \\
\hline & $\begin{array}{l}27,6 \\
28,4 \\
25,5 \\
\\
26,2\end{array}$ & $\begin{array}{r}19,0 \\
18,0 \\
2,5\end{array}$ & & & $\begin{array}{c}9 \text { meses } \\
18,0 \\
10,0 \\
7,5\end{array}$ & $\begin{array}{l}\text { Autoinforme. } \\
\text { Tiocianato y } \\
\text { cotinina de } \\
\text { una pequeña } \\
\text { muestra. }\end{array}$ \\
\hline $\begin{array}{l}\text { - Residir en Galicia. } \\
\text { - Ser fumador de cigarrillos. } \\
\text { - Fumar mínimo } 10 \text { cigar./ } \\
\text { - día } \\
\text { - No usar terapia farmaco- } \\
\text { lógica ni psicoterapia. } \\
\text { - Querer tratamiento aun- } \\
\text { que se retrase } \\
\text { - Rellenar cuestionario. } \\
\text { - Enviar autoevaluación. } \\
\text { - Firmar consentimiento. }\end{array}$ & & $\begin{array}{r}37,0 \\
1,0\end{array}$ & $\begin{array}{r}32,0 \\
1,0\end{array}$ & 13,0 & $\begin{array}{l}51,0 \\
0,0\end{array}$ & $\begin{array}{l}\text { Autoinforme y } \\
\text { CO (a los } 6 \text { y } \\
12 \text { meses) }\end{array}$ \\
\hline
\end{tabular}

Se envió la primera lección del curso dentro de las dos semanas de haber devuelto el formulario de registro. Las lecciones siguientes fueron enviadas en intervalos de una semana. Cinco días después de que la última lección fuera enviada, se entrevistaba por teléfono a los participantes y a los sujetos control. Antes del curso, se tomaran muestras de saliva de 31 sujetos, seleccionados aleatoriamente, para un análisis del tiocianato. Después del curso, 25 de ellos repitieron la evaluación.

Se contactó con los participantes por teléfono, 3 y 9 meses después de con- cluido el tratamiento. En el seguimiento del noveno mes se recogieron muestras de orina para evaluar la cotinina. En cuanto a la abstinencia, el porcentaje obtenido en el curso personalizado fue más alto al final del tratamiento $(94 \%$ vs. $58 \%$ ). Sin embargo, no había diferencias estadísticamente significativas entre los grupos a los tres meses ( $76 \%$ vs. $58 \%$ ) 6 9 meses de seguimiento ( $41 \%$ vs. $54 \%$ ). Si se consideran a todos los no localizados como fumadores, el porcentaje de abstinencia al final del tratamiento para el grupo control es del 2.5\%, para el curso estándar $19 \%$ y para el curso persona- 
lizado $22 \%$. Por lo que respecta a la abstinencia a los tres meses, ésta pasó a ser de $2.5 \%, 19 \%$ y $18 \%$, respectivamente. A los nueve meses de seguimiento, fue del $7.5 \%, 18 \%$ y $10 \%$ para cada grupo.

En este caso, la versión personalizada del curso, adaptada a las características de cada fumador, no resultó en un incremento de la efectividad, como había sugerido previamente Schneider et al. (1984). Owen et al. (1989) consideraron que quizás los elementos personalizados, tal y como estaban implantados en el texto, pudieron no haber sido suficientemente distintivos. De ahí que sugieran que, futuras investigaciones, deberían usar un formato donde toda la información personalizada se presente en una carta que acompañe a las lecciones estándar. Esto podría aumentar la distintividad del material personalizado y resultaría de mayor facilidad de elaboración, respecto de una lección completa en formato personalizado.

En la línea de lo anterior, en España, Becoña y Vázquez (2001b) evaluaron los efectos de la utilización de feedback escrito personalizado en una intervención de autoayuda por correo. La muestra utilizada fue de 300 sujetos asignados aleatoriamente a tres condiciones: 1) tratamiento estándar (manual) $(n=100) ; 2)$ tratamiento estándar más feedback escrito personalizado ( $n=100) ;$ y 3) grupo control de lista de espera $(n=100)$. Ambos tratamientos, estándar y personalizado, duraban seis semanas. El feedback escrito personalizado consistía en dos envíos, de una página de extensión, elaborados en función de las respuestas que los fumadores daban a las autoevaluaciones semanales. El primero de ellos fue enviado entre la tercera y cuarta semana de tratamiento $y$, el segundo, entre la quinta y sexta. El grupo control no recibió tratamiento hasta 6 meses después de finalizar el tratamiento los dos grupos experimentales.
Al final del tratamiento y en el seguimiento del tercer mes, para evaluar la abstinencia, se entrevistó a los participantes por teléfono o correo. A los 6 y 12 meses de seguimiento se verificaron bioquímicamente, a través de monóxido de carbono en aire espirado, los autoinformes de abstinencia. La eficacia del tratamiento por correo estándar fue del $37 \%$ al final del tratamiento, $22 \%$ a los 3 meses de seguimiento, $19 \%$ a los 6 meses y $13 \%$ a los 12 meses. El grupo que recibió feedback escrito personalizado obtuvo una eficacia del $51 \%$ al final del tratamiento, $37 \%$ a los 3 meses de seguimiento, $32 \%$ a los 6 meses y $27 \%$ a los 12 meses. Ambas intervenciones, pues, obtuvieron un porcentaje de éxito importante. Además, el feedback escrito personalizado incrementó el porcentaje de abstinencia en todos los momentos temporales. Los autores estiman que el método de aplicación del feedback individualizado que utilizaron, dentro de la intervención de autoayuda, resultó ser un aspecto clave. En este caso, se llevó a cabo adaptando las recomendaciones de Miltenberger (1997) para proporcionar feedback en el contexto clínico.

\section{DISCUSIÓN}

La diversidad de factores que concurren en las diferentes investigaciones revisadas, en cuanto a muestras utilizadas, criterios de admisión y criterios de abstinencia, entre otros, dificulta su comparación. Aún así, a nivel general, los estudios muestran como los porcentajes de abstinencia obtenidos mediante intervenciones de autoayuda por correo son inferiores a los obtenidos con programas clínicos debido, básicamente, a la inexistencia de contacto terapéutico y menor adherencia que en un programa clínico. Sin embargo, se deben tener en cuenta limitaciones, a nivel metodológico, que 
en parte de estos estudios han dificultado la evaluación de la eficacia de tal estrategia de abstinencia del tabaco. Una de ellas, hace referencia a la corta duración de los seguimientos, en algunos casos inferiores al año (p.ej., Lando et al., 1991; Owen et al., 1989), cuando lo que se recomienda es hacer seguimientos hasta el año de finalizado el tratamiento (Lando, 1989,1993; Velicer, Prochaska, Rossi y Snow., 1992). De hecho, varios estudios no fueron incluidos en este trabajo (p.ej., Ershoff, Mullen y Quinn, 1989; Jeffery, Hellersted y Schmid, 1990) por presentar seguimientos de menos de seis meses. Además de esto, algunos de los estudios revisados, en la valoración de los resultados obtenidos, se basaron exclusivamente en los autoinformes de los sujetos (p.ej., Davis et al., 1984; Lando et al., 1991), sin llevar a cabo verificación bioquímica de la abstinencia. Esto implica que los datos dependen de la honestidad y exactitud de la información proporcionada por los participantes.

Es también característico que, en general, se proporcione poca información en relación al procedimiento de intervención utilizado, lo cual dificulta enormemente la posibilidad de replicación.

Un dato que a primera vista puede resultar curioso, que se observa en intervenciones de autoayuda por correo (p.ej., Davis et al, 1984), es que el porcentaje de abstinencia aumenta a lo largo de los seguimientos. Ello puede deberse al incremento en los intentos de abandono por parte de reincidentes previos. Este dato apoya la idea de que un patrón típico en los fumadores que no buscan tratamiento, es un repetido intento para dejar de fumar, con períodos de abstinencia a los que siguen otros de recaida (Gil y Calero, 1994). Otra posible explicación es que el porcentaje de fumadores que termina un programa de autoayuda se incrementa con el tiempo.

Destaca el que varios factores que dis- tinguen a los estudios revisados pueden haber influido, en mayor o menor medi$\mathrm{da}$, en los resultados obtenidos. Por ejemplo, las estrategias de reclutamiento utilizadas, con el objetivo de llegar a subgrupos de fumadores que no tienden a inscribirse en programas de tratamiento. Los procedimientos más utilizados son los anuncios en la prensa y/o medios de comunicación (Becoña y Vázquez, 2001a, 2001b; Davis et al., 1984; Owen et al., 1989) o el marcar números telefónicos aleatoriamente para identificar a los fumadores a través de una encuesta poblacional (Lando et al., 1991). El criterio de reclutamiento que se utilice en una intervención de autoayuda es un aspecto importante, puesto que las intervenciones dirigidas a la totalidad de la población tienen un mayor impacto que aquellas que se centran en poblaciones de voluntarios (Velicer, Rossi, Ruggiero y Prochaska, 1994). Algunos autores (p.ej., Wagner, Shoenbach, Orleans et al., 1990) han llegado a sugerir que esos voluntarios que solicitan tratamiento pueden no ser los mejores candidatos para este tipo de intervenciones mínimas (p.ej., suelen tener más edad, son más adictos, su momento de abandono es más duro o difícil, están menos seguros de sí mismos con respecto al abandono del tabaco y tienen menos apoyo social que la población general de fumadores). Por otro lado, se ha visto que la distribución de materiales de autoayuda a no voluntarios no produce cambios significativos (p.ej., Lando et al., 1991). Por lo tanto, el hecho de haber utilizado voluntarios o no voluntarios es un factor a tener en cuenta, pues presuntamente la motivación no es la misma. En una muestra de voluntarios, la mayoría de los sujetos están preparados para emprender la acción de modificar un problema de conducta. Sin embargo, en una muestra reclutada dentro de la población general, un amplio número de sujetos, o no se han planteado cambiar, o están empezan- 
do a pensar en ello. Teniendo en cuenta que ambas muestras son diferentes, la intervención que resulta más apropiada para voluntarios, puede no resultar apropiada para la población general de fumadores, en la que suele estar guiada por el modelo transteórico de cambio.

La diferencia en los criterios de admisión es otra variable de los estudios que también influye en la selección que se haga de los participantes (ver Tablas 1 y 2). Así, tenemos estudios que no imponen criterio alguno (p.ej., Lando et al., 1991; Owen et al., 1989), y otros que marcan hasta ocho criterios de inclusión (p.ej., Becoña y Vázquez, 2001b).

Una de las cuestiones que preocupa particularmente en estas intervenciones es saber si se da una relación dosis-respuesta entre el uso de los materiales y el abandono del tabaco. De hecho, una variable que resulta de gran importancia, para interpretar los resultados, es el grado de uso que se haya hecho de los materiales, puesto que es un factor que se ha asociado a porcentajes de abstinencia más altos, llegándose incluso a afirmar (Curry, 1993; Míguez, Vázquez y Becoña, 2000) que los porcentajes de abstinencia para los fumadores que utilizan materiales de autoayuda (p.ej., siguen activamente las actividades sugeridas, no limitándose simplemente a leer el manual) suelen ser comparables a los obtenidos por los fumadores que participan en programas en grupo más intensivos. El uso de los materiales, a diferencia de sólo su lectura, se ha asociado con porcentajes de abstinencia más altos tanto a corto (p.ej., Curry et al., 1991; Gritz et al., 1992; Míguez et al., 2000) como a largo plazo (p.ej., Davis et al, 1984; Gritz, Berman, Bastani y Wu, 1992; Míguez et al., 2000). De hecho, los manuales de autoayuda se han mostrado facilitadores del cambio de conducta, especialmente si resulta posible incrementar la ejecución de su contenido, pues suele darse una baja adherencia a los mismos.
En cuanto al efecto de determinadas características del material de autoayuda, tales como su extensión y/o forma de entrega (manual/folletos y envío único/envíos semanales), no parecen constituir una variable decisiva, aun cuando habría que diferenciar entre folletos informativos y folletos con el mismo contenido que el manual (p.ej., Becoña y Vázquez, 2001a), pues puede tratarse de una variable clave $y$, sin embargo, los estudios no siempre informan de este dato. Además, en este caso, sería necesario valorar otros aspectos, como los costes del tratamiento; hay que tener en cuenta que la confección y envío de folletos resulta más barata que la de un manual.

Como ya hemos indicado, una de las principales críticas que se achacan a las intervenciones de autoayuda por correo para dejar de fumar es su impersonalización. Se han utilizado diferentes estrategias para personalizar estas intervenciones y favorecer una mayor adherencia al tratamiento. El feedback escrito personalizado tiene como finalidad, entre otras, el aumentar la confianza en dejar de fumar (Curry, Louie, Grothaus y Wagner, 1992). Se trata de una forma alternativa de proporcionar ayuda personalizada, sin recurrir al consejo cara a cara. Los resultados de los estudios que utilizan tal procedimiento no son del todo concluyentes. Por ejemplo, Owen et al. (1989) informan que con la personalización del material no consiguieron mejorar los resultados. Sin embargo, Becoña y Vázquez (2001b) hallaron que el grupo que recibió feedback escrito personalizado obtuvo mejores resultados en todos los momentos temporales. No existen suficientes datos para poder determinar qué elementos de la personalización pueden ser los más importantes (Strecher, 1999), aunque algunos autores (p.ej., Becoña y Vázquez, 2001b) indican que la clave en el feedback personalizado se encuentra en el método que se utilice para la apli- 
cación del feedback, pues los procedimientos utilizados son diferentes de unos estudios a otros.

Quedan todavía muchas cuestiones por analizar en este tipo de intervenciones. Una de ellas hace referencia a si la participación en un contexto de autoayuda requiere más auto-motivación, puesto que lo habitual es que no implique contacto personal con el terapeuta (Sussman, Dent, Wang et al., 1994). En este caso, el cliente tiene que convertirse en su propio terapeuta. Resultaría interesante poder establecer si determinadas características de los fumadores están relacionadas con el éxito de una intervención de autoayu$\mathrm{da}$, bien porque incrementen la probabilidad de uso de algunos materiales de autoayuda (p.ej., nivel educativo), bien por interacciones entre características de los participantes y diferentes intervenciones, es decir, que algunas funcionen mejor para un determinado tipo de fumadores. Investigaciones futuras deberían centrarse en identificar el tipo de fumadores que con mayor probabilidad se beneficiaría de una determinada modalidad de intervención de autoayuda, pues permitiría su optimización.

Nos encontramos ante una alternativa más de tratamiento para los fumadores, de las múltiples existentes, para poder intervenir sobre la primera causa de morbi-mortalidad de los países desarrollados. Los diferentes abordajes terapéuticos que existen para el tratamiento del tabaquismo deben complementarse y estar a disposición de todos los fumadores, para que cada cual elija el que se adapte mejor a sus necesidades. Los procedimientos de autoayuda son uno de ellos.

\section{REFERENCIAS BIBLIOGRÁFICAS}

American Lung Association (1984). Freedom from smoking in 20 days. Washington, DC: Author.
American Lung Association (1986). A lifetime of freedom from smoking: A maintenance program for ex-smokers. New York: Author.

Becoña, E. (1993). Programa para dejar de fumar. Santiago de Compostela: Servicio de Publicaciones e Intercambio Científico de la Universidad de Santiago de Compostela

Becoña, E. (1994). Tratamiento del tabaquismo. En J.L. Graña (Ed.), Conductas adictivas: teoría, evaluación y tratamiento (pp. 455-493). Madrid: Debate.

Becoña E., y Vázquez, F.L. (1998). Tratamiento del tabaquismo. Madrid: Dykinson.

Becoña E., y Vázquez, F.L. (2001a). Comparación de la eficacia de un programa de autoayuda para dejar de fumar distribuido por correo en un único envío o semanalmente en folletos. Psiquis, 4, 205-214.

Becoña E., y Vázquez, F.L. (2001b). Effectiveness of personalized written feedback through a mail intervention for smoking cessation: A randomized-controlled trial in Spanish smokers. Journal of Consulting and Clinical Psychology, 69, 33-40.

Brown, S.L., Hunt, G., y Owen, N. (1992). The effect of adding telephone contact to selfinstructional smoking-cessation materials. Behaviour Change, 9, 216-222.

Brown, S.L., y Owen, N. (1992). Self-help smoking cessation materials. Australian Journal of Public Health, 16, 188-191

Cohen, S., Lichtenstein, E., Prochaska, J.O., Rossi, J.S., Gritz, E.R., Carr, C.R., Orleans, C.T., Schoenbach, V.J., Biener, L., Abrams, D., DiClemente, C., Curry, S., Marlatt, G.A., Cummings, K.M., Emont, S.L., Giovino, G., Ossip-Klein, D. (1989). Debunking myths about self-quitting. Evidence from 10 prospective studies of persons who attempt to quit smoking by themselves. American Psychologist, 44, 1355-1365.

Cummings, K.M., Emont, S.L., Jaen, C., y Sciandra, R. (1988). Format and quitting instructions as factors influencing the impact of a self-administered quit smoking program. Health Education Quarterly, 15, 199-216.

Cummings, K.M., Sciandra, R., Davis, S., y Rimer, B. (1989). Response to anti-smoking campaign aimed at mothers with young 
children. Health Education Research Theory and Practice, 4, 429-437.

Curry, S.J. (1993). Self-help interventions for smoking cessation. Journal of Consulting and Clinical Psychology, 61, 790-803.

Curry, S.J. (2001). Bridging the clinical and public health perspectives in tobacco treatment research: Scenes from a tobacco treatment research carer. Cancer Epidemiology Biomarkers and Prevention, 10, 281-285.

Curry, S.J., Louie, D., Grothaus, L.C., y Wagner, E.H. (1992). Written personalized feedback and confidence for smoking cessation. Psychology of Addictive Behaviors, 6, 175-180.

Curry, S.J., Wagner, E.H., y Grothaus, L.C. (1991). Evaluation of intrinsic and extrinsic motivation interventions with a self-help smoking cessation program. Journal of Consulting and Clinical Psychology, 59, 318-324.

Danaher, B.G., y Lichtenstein, E. (1978) Become and ex-smoker. Englewood Cliffs, N.J.: Prentice Hall.

Davis, S.W., Cummings, K.M., Rimer, B.K., Sciandra, R., Stone, J.C. (1992). The impact of tailored self-help smoking cessation guides on young mothers. Health Education Quarterly, 19, 495-504.

Davis, A.L., Faust, R., y Ordentlich, M. (1984). Self-help smoking cessation and maintenance programs: A comparative study with 12-month follow-up by the American Lung Association. American Journal of Public Health, 74, 1212-1217.

Dijkstra, A., De Vries, H., Roijackers, J., y Breukelen, G. (1998a). Tailored interventions to communicate stage-matched information to smokers in different motivational stages. Journal of Consulting and Clinical Psychology, 66, 549-557.

Dijkstra, A., De Vries, H., Roijackers, J., y Breukelen, G. (1998b). Tailoring information to enhance quitting in smokers with low motivation to quit: Three basic efficacy questions. Health Psychology, 17, 1-7.

Ershoff, D.H., Mullen, P.D., y Quinn, V.P. (1989). A randomized trial of a serialized self-help smoking cessation program for pregnant women in an HMO. American Journal of Public Health, 79, 182-187.

Fiore, M.C., Novotny, T.E., Pierce, J.P., Giovino, G.A. (1990). Methods used to quit smo- king in the United States: do cessation programs help?. Journal of the American Medical Association, 263, 2760-2765.

Gil, J., y Calero, M.D. (1994). Tratamiento del tabaquismo. Madrid: McGraw-Hill-Interamericana de España.

Glasgow, R.E., Schafer, L., y O'Neill, H.K. (1981). Self-help books and amount of therapist contact in smoking cessation programs. Journal of Consulting and Clinical Psychology, 49, 659-667.

Glynn, T.J., Boyd, G.M., y Gruman, J.C. (1990a). Essential elements of selfhelp/minimal intervention strategies for smoking cessation. Health Education Quarterly, 17, 329-345.

Glynn, T.J., Boyd, G.M., y Gruman, J.C. (1990b). Self-guided strategies for smoking cessation: A Program Planner's Guide. Rockville, MD: U.S. Department of Health and Human Services.

Gritz, E.R., Berman, B.A., Bastani, R., y Wu, $M$. (1992). A randomized trial of a selfhelp smoking cessation intervention in a nonvolunteer female population: Testing the limits of the public health model. Health Psychology, 11, 280-289.

Jeffery, R.W., Danaher, B.G., Killen, J., Farquhar, J.W., y Kinnier, R. (1982). Self-administered programs for health behavior change: Smoking cessation and weight reduction by mail. Addictive Behaviors, 7, 57-63.

Jeffery, R.W., Hellerstedt, W.L., y Schmid, T.L. (1990). Correspondence programs for smoking cessation and weight control: A Comparison of two strategies in the Minnesota Heart Health Program. Health Psychology, 9, 585-598.

Lancaster, T., y Stead, L.F. (2002). Self-help interventions for smoking cessation. (Cochrane Review). En The Cochrane Library, 3. Oxford: Update Software.

Lando, H.A. (1989). Treatment outcome evaluation methodology in smoking cessation: Strengths and key issues. Advances in Behavior Research and Therapy, 11, 201214.

Lando, H.A. (1993). Formal Quit Smoking treatments. En C.T. Orleans y J. Slade. Nicotine addiction principles and management (pp. 221-244) New York: Oxford University Press. 
Lando, H.A., Pirie, P.L., McGovern, P.G., Pechacek, T.F., Swim, J., Loken, B. (1991). A comparison of self help approaches to smoking cessation. Addictive Behaviors, 16, 183-193.

Lichtenstein, E. (1982). The smoking problem: A behavioral perspective. Journal of Consulting and Clinical Psychology, 50, 804-819.

Lichtenstein, E., y Glasgow, R.E. (1992). Smoking cessation: What have we learned over the past decade?. Journal of Consulting and Clinical Psychology, 60, 518-527.

Miltenberger, R. (1997). Behavior modification. Principles and procedures. Pacific Grove, CA: Brooks/Cole.

Míguez, M.C., Vázquez, F.L., y Becoña, E. (2000). Realización de las tareas en un tratamiento para dejar de fumar y sus repercusiones clínicas. Psicología Contemporánea, 7, 32-35.

Míguez, M.C., Vázquez, F.L., y Becoña, E. (2002). Effectiveness of telephone contact as an adjunct to a self-help program for smoking cessation. A randomized controlled trial in Spanish smokers. Addictive Behaviors, 27, 139-144.

National Cancer Institute (1985). Quit for Good. Rockville, MD: U.S. Department of Health and Human Services.

O'Farrell, T. J., y Keuthen, N.J. (1983). Readability of behavior therapy self-help manuals. Behavior Therapy, 14, 449-455.

Orleans, C.T., Schoenbach, V.J, Wagner, E.H., Quade, D., Salmon, M.A., Pearson, D.C., Fiedler, J., Porter, C.Q., y Kaplan, B.H. (1991). Self-help quit smoking interventions: Effects of self-help materials, social support instructions and telephone counselling. Journal of Consulting and Clinical Psychology, 59, 439-448.

Owen, N., Ewins, A.L., y Lee, C. (1989). Smoking cessation by mail: A comparison of standard and personalized correspondence course formats. Addictive Behaviors, 14, 355-363.

Pechacek, T.F., Arkin, R., y Johnston Hall, N. (1984). Quit and Win. Minneapolis, MI: University of Minnesota, Minnesota Heart Health Program.

Prochaska, J.O., DiClemente, C.C., Velicer, W.F., y Rossi, J.S. (1993). Standarized, individualized, interactive, and personalized self-help programs for smoking cessation. Health Psychology, 12, 399-405.

Prochaska, J.O., y Procheska, J.M. (1993). Modelo transteórico de cambio para conductas adictivas. En M. Casas y M. Gossop (coord.), Recaída y prevención de recaídas. Tratamientos psicológicos en drogodependencias (pp. 85-136). Barcelona: Ediciones en Neurociencias.

Rimer, B.K., Orleans, C.T., Fleisher, L., Cristinzio, S., Resch, N., Telepchak, J., y Keintz, M.K. (1994). Does tailoring matter? The impact of a tailored guide on ratings and short-term smoking-related outcomes for older smokers. Health Education Research, 9, 69-84.

Schneider, S.J., Benya, A., y Singer, H. (1984). Computerized direct mail to treat smokers who avoid treatment. Computers and Biomedical, 17, 409-418.

Schwartz, J.L. (1987). Review and evaluation of smoking cessation methods. The United States and Canada, 1978-1985. Rockville, MD: U.S. Department of Health and Human Services. National Institutes of Health.

Shiffman, S., Paty, J.A., Rohay, J.M., Di-Marino, M.E., y Gitchell, J. (2000). The efficacy of computer-tailored smoking cessation material as a supplement to nicotine polacrilex gum therapy. Archives of Internal Medicine, 160, 1675-1681.

Strecher, V.J. (1999). Computer-tailored smoking cessation materials: A review and discussion. Patient Education and Counselling, 36, 107-117.

Strecher, V.J., Kreuter, M., Den Boer, D.J., Kobrin, S., Hospers, H.J., Skinner, C.S. (1994). The effects of computer-tailored smoking cessation messages in family practice settings. Journal of Family Practice, 39, 262-270.

Strecher, V.J., Rimer, B.K., y Monaco, K.D. (1989). Development of a new self-help guide-Freedom from Smoke for You and Your Family. Health Education Quarterly, 16, 101-112.

Sussman, S., Dent, C.W., Wang, E., Cruz, N.T.B., Stanford, D., y Johnson, C.A. (1994). Participants and nonparticipants of a mass media self-help smoking cessation program. Addictive Behaviors, 19, 643654. 
U.S.D.H.H.S. (1988). The health consequences of smoking: Nicotine addiction. A Report of the Surgeon General. Rockville, MD: U.S. Department of Health Human Services, Office on Smoking and Health. Velicer, W.F., Prochascha, J.O., Rossi, J. S., y Snow, M. (1992). Assessing outcome in smoking cessation studies. Psychological Bulletin, 111, 23-41

Velicer, W.F., Rossi, J.S., Ruggiero L., y Prochaska, J.O. (1994). Minimal interventions appropriate for an entire population of smokers. En R. Richmond (Ed.), Interventions for smokers: An international perspective. Baltimore: Williams and Wilkins.

Wagner, E.H., Shoenbach, V.J., Orleans, C.T., Grothaus, L.C., Saunders, K.W., Curry, S.J., y Pearson, D.C. (1990). Participation in a smoking cessation program: A populationbased perspective. American Journal of Preventive Medicine, 6, 258-266.

Windsor, R.A., Lowe, J.B., Perkins, L.L., Smith-Yoder, D., Artz, L., Crawford, M., Amburgy, K., y Boyd, N.R. (1993). Health education for pregnant smokers: Its behavioral impact and cost benefit. American Journal of Public Health, 83, 201-206. 